\title{
A numerical code for the solution of the Kompaneets equation in cosmological context
}

\author{
P. Procopio ${ }^{1,2}$ and C. Burigana ${ }^{1,2}$ \\ 1 INAF/IASF-BO, Istituto di Astrofisica Spaziale e Fisica Cosmica di Bologna, Via Gobetti 101, 40129 Bologna, Italy \\ e-mail: burigana@iasfbo.inaf.it \\ 2 Dipartimento di Fisica, Università degli Studi di Ferrara, Via Saragat 1, 44100 Ferrara, Italy
}

Received 13 March 2009 / Accepted 23 July 2009

\begin{abstract}
Context. After fundamental ground-based, balloon-born, and space experiments, and, in particular, after the COBE/FIRAS results, confirming that only very small deviations from a Planckian shape can be present in the CMB spectrum, current and future CMB absolute temperature experiments aim at discovering very small distortions such as those associated with the cosmological reionization process or that could be generated by different kinds of earlier processes.

Aims. Interpretation of future data calls for a continuous improvement in the theoretical modeling of CMB spectrum. In this work we describe the fundamental approach and, in particular, the update to recent NAG versions of a numerical code, KYPRIX, specifically written to solve the Kompaneets equation in a cosmological context. It was first implemented in the years 1989-1991 to accurately compute the CMB spectral distortions under general assumptions.

Methods. Specifically, we describe the structure and the main subdivisions of the code and discuss the most relevant aspects of its technical implementation. After a presentation of the equation formalism and of the boundary conditions added to the set of ordinary differential equations derived from the original parabolic partial differential equation, we provide details on the adopted space variable (i.e. dimensionless frequency) and space discretization, on time variables, on the output results, on the accuracy parameters, and on the used auxiliary integration routines. The problem with introducing the time dependence of the ratio between electron and photon temperatures and of the radiative Compton scattering term, both of them introducing integral terms into the Kompaneets equation, is addressed in the specific context of the recent NAG versions. We describe the introduction of the cosmological constant in the terms controlling the general expansion of the Universe in agreement with the current concordance model, of the relevant chemical abundances, and on the ionization history, from recombination to cosmological reionization. The global computational time, the impact of the various aspects of the code on it, and the accuracy of the numerical integration are also discussed.

Results. We present some of fundamental tests we carried out to verify the accuracy, reliability, and performance of the code. We focus on some quantitative tests of energy conservation and the time behavior of electron temperature. A comparison of the results obtained with the update and the original version of the code is presented for some representative cases. Finally, we focus on some properties of the free-free distortions relevant for the long wavelength region of the CMB spectrum.

Conclusions. All the tests demonstrate the reliability and versatility of the new code version and its accuracy and applicability to the scientific analysis of current CMB spectrum data and of much more precise measurements that will be available in the future. The recipes and tests described in this work can also be useful for implementing accurate numerical codes for other scientific purposes using the same or similar numerical libraries or for verifying the validity of different codes aimed at the same problem or similar ones.
\end{abstract}

Key words. cosmic microwave background - radiation mechanisms: general - radiative transfer - scattering - methods: numerical

\section{Introduction}

The CMB spectrum emerges from the thermalization redshift, $z_{\text {therm }} \sim 10^{6}-10^{7}$, with a shape very close to Planckian, owing to the tight coupling between radiation and matter through Compton scattering and photon production/absorption processes, radiative Compton, and bremsstrahlung. These processes were extremely efficient at early times and able to reestablish a blackbody (BB) spectrum from a perturbed one on much shorter timescales than the expansion time (see e.g. Danese \& de Zotti 1977). The value of $z_{\text {therm }}$ (Burigana et al. 1991a) depends on the baryon density parameter, $\Omega_{\mathrm{b}}$, and the Hubble constant, $H_{0}$, through the product $\widehat{\Omega}=\Omega_{\mathrm{b}}\left(H_{0} / 50\right)^{2}\left(H_{0}\right.$ expressed in $\mathrm{km} \mathrm{s}^{-1} \mathrm{Mpc}^{-1}$ ).

On the other hand, physical processes occurring at redshifts $z<z_{\text {therm }}$ may leave imprints on the CMB spectrum. Therefore, the CMB spectrum carries crucial information on physical processes occurring during early cosmic epochs (see e.g. Danese \& Burigana 1994 and references therein), and the comparison between models of CMB spectral distortions and CMB absolute temperature measures can constrain the physical parameters of the considered dissipation processes (Burigana et al. 1991b).

The timescale for the achievement of kinetic equilibrium between radiation and matter (i.e. the relaxation time for the photon spectrum), $t_{\mathrm{C}}$, is

$t_{\mathrm{C}}=t_{\gamma \mathrm{e}} \frac{m_{\mathrm{e}} c^{2}}{k T_{\mathrm{e}}} \simeq 4.5 \times 10^{28}\left(T_{0} / 2.7 \mathrm{~K}\right)^{-1} \phi^{-1} \widehat{\Omega}^{-1}(1+z)^{-4} \mathrm{~s}$,

where $t_{\gamma \mathrm{e}}=1 /\left(n_{\mathrm{e}} \sigma_{\mathrm{T}} c\right)$ is the photon-electron collision time, $T_{\mathrm{e}}$ and $T_{\mathrm{r}}=T_{0}(1+z)$ are respectively the electron and the CMB radiation temperature, $\phi=\left(T_{\mathrm{e}} / T_{\mathrm{r}}\right) ; k T_{\mathrm{e}} / m_{\mathrm{e}} c^{2}$ (with $m_{\mathrm{e}}$ the electron mass) is the mean fractional change of photon energy in a 
scattering of cool photons off hot electrons, i.e. $T_{\mathrm{e}} \gg T_{\mathrm{r}} ; T_{0}$ is the present radiation temperature related to the present radiation energy density by $\epsilon_{r 0}=\mathrm{a} T_{0}^{4}$ (here a $\left.=8 \pi I_{3} k^{4} /(h c)^{3}, I_{3}=\pi^{4} / 15\right)$. A primordial helium abundance of $25 \%$ in mass is assumed in these numerical estimates. It is useful to introduce the dimensionless time variable $y_{\mathrm{e}}(z)$ defined by

$y_{\mathrm{e}}(z)=\int_{t}^{t_{0}} \frac{\mathrm{d} t}{t_{\mathrm{C}}}=\int_{1}^{1+z} \frac{\mathrm{d}(1+z)}{1+z} \frac{t_{\mathrm{exp}}}{t_{\mathrm{C}}}$,

where $t$ is the time, $t_{0}$ is the present time, and $t_{\exp }=1 / H=$ $1 /[(\mathrm{d} a / \mathrm{d} t) / a]$ is the expansion time, and where $a=1 /(1+z)$ is the cosmic scale factor normalized to the present time.

In particular, by neglecting the cosmological constant (or dark energy) contribution, we have

$$
\begin{aligned}
& t_{\exp } \simeq 6.3 \times 10^{19}\left(\frac{T_{0}}{2.7 \mathrm{~K}}\right)^{-2}(1+z)^{-3 / 2} \\
& \times\left[\kappa(1+z)+\left(1+z_{\mathrm{eq}}\right)-\left(\frac{\Omega_{\mathrm{nr}}-1}{\Omega_{\mathrm{nr}}}\right)\left(\frac{1+z_{\mathrm{eq}}}{1+z}\right)\right]^{-1 / 2} \mathrm{~s},
\end{aligned}
$$

where $z_{\mathrm{eq}}=1.0 \times 10^{4}\left(T_{0} / 2.7 \mathrm{~K}\right)^{-4} \widehat{\Omega}_{\mathrm{nr}}$ is the redshift of equal non relativistic matter and photon energy densities and $\kappa=$ $1+N_{v}(7 / 8)(4 / 11)^{4 / 3}$ takes into account the contribution of relativistic neutrinos to the dynamics of the Universe ${ }^{1}$; here $N_{v}$ is the number of relativistic, 2-component, neutrino species (for 3 species of massless neutrinos, $\kappa \simeq 1.68$ ). While assuming $\Omega_{\mathrm{K}}=0, \Omega_{\Lambda}=1-\Omega_{\mathrm{nr}}$, and neglecting the radiation energy density, as possible at relatively low redshifts, we have

$t_{\exp } \simeq\left(1 / H_{0}\right)\left[\Omega_{\mathrm{nr}}(1+z)^{3}+1-\Omega_{\mathrm{nr}}\right]^{-1 / 2} \mathrm{~s}$,

where $1 / H_{0} \simeq 3.1 \times 10^{17} h^{-1} \mathrm{~s}\left(h=H_{0} / 100\right)$.

The time evolution of the photon occupation number, $\eta(v, t)$, under the combined effect of Compton scattering and of photon production processes, namely radiative Compton (RC) (Gould 1984), bremsstrahlung (B) (Karzas \& Latter 1961; Rybicki \& Lightman 1979), plus other possible photon emission/absorption contributions (EM) ${ }^{2}$, is described well by the complete Kompaneets equation (Kompaneets 1956; Burigana et al. 1995):

$$
\begin{aligned}
\frac{\partial \eta}{\partial t}= & \frac{1}{\phi} \frac{1}{t_{\mathrm{C}}} \frac{1}{x^{2}} \frac{\partial}{\partial x}\left[x^{4}\left[\phi \frac{\partial \eta}{\partial x}+\eta(1+\eta)\right]\right] \\
& +\left[\frac{\partial \eta}{\partial t}\right]_{\mathrm{RC}}+\left[\frac{\partial \eta}{\partial t}\right]_{\mathrm{B}}+\left[\frac{\partial \eta}{\partial t}\right]_{\mathrm{EM}} .
\end{aligned}
$$

This equation is coupled to the time differential equation governing the electron temperature evolution for an arbitrary radiation

\footnotetext{
${ }^{1}$ Strictly speaking the present ratio of neutrino to photon energy densities, hence the value of $\kappa$, is itself a function of the amount of energy dissipated. The effect, however, is never very strong and is negligible for very small distortions.

2 A process that should be included is the cyclotron emission. On the other hand, for realistic values of cosmic magnetic field, the cyclotron process never plays an important role for (global) CMB spectral distortions when ordinary and stimulated emission and absorption are properly taken into account and CMB realistic distorted spectra are considered (Zizzo \& Burigana 2005). In fact, the cyclotron term may be significant in the case of deviations of $\eta$ from the BB distribution at the electron temperature, only at very long wavelengths, corresponding to the cyclotron frequency, where, during the formation of a spectral distortion, FF and RC are able to keep $\eta$ extremely close to the $\mathrm{BB}$ equilibrium.
}

spectrum in the presence of Compton scattering, energy losses due to radiative Compton and bremsstrahlung, adiabatic cooling, plus possible external heating sources, $q=a^{-3}(\mathrm{~d} Q / \mathrm{d} t)$,

$$
\frac{\mathrm{d} T_{\mathrm{e}}}{\mathrm{d} t}=\frac{T_{\mathrm{eq}, \mathrm{C}}-T_{\mathrm{e}}}{(27 / 28) t_{\mathrm{e} \gamma}}-\frac{2 T_{\mathrm{e}}}{t_{\mathrm{exp}}}+\left[\frac{\mathrm{d} T_{\mathrm{e}}}{\mathrm{d} t}\right]_{\mathrm{RC}, \mathrm{B}}+\frac{(32 / 27) q}{3 n_{\mathrm{e}} k} .
$$

Here, $T_{\text {eq,C }}=\left[h \int \eta(1+\eta) v^{4} \mathrm{~d} v\right] /\left[4 k \int \eta v^{3} \mathrm{~d} v\right]$ is the Compton equilibrium electron temperature (Peyraud 1968; Zeldovich \& Levich 1970), $t_{\mathrm{e} \gamma}=3 m_{\mathrm{e}} c / 4 \sigma_{\mathrm{T}} \epsilon_{r}, \epsilon_{r} \simeq \epsilon_{r 0}(1+z)^{4}$ with $\epsilon_{r 0}=a T_{0}^{4}$ the radiation energy density today, and $x=h v_{0} / k T_{0}=h v_{0}(1+$ $z) / k T_{0}(1+z)$ is a dimensionless, redshift-independent frequency ( $v_{0}$ being the present frequency).

\section{Setting up the problem}

Partial differential linear equations are divided into three classes: elliptic, parabolic, and hyperbolic. The Kompaneets equation is a parabolic partial differential equation (Tricomi 1957). Solutions to this equation under general conditions have to be searched numerically, because it is impossible to find analytical solutions that accurately take the many kinds of cosmological scenarios and the great number of relevant physical processes into account. The numerical code KYPRIX was written to overcome the limited applicability of analytical solutions and to get a precise computation of the evolution of the photon distribution function for a wide range of cosmic epochs and for many cases of cosmological interest (Burigana et al. 1991a). KYPRIX makes use of the NAG libraries (NAG Ltd 2009).

Besides these libraries, a lot of numerical algorithms are used in the code: we used some of the routines available to the scientific community, but often we wrote routines dedicated to a specific task. Among the formers, the D03PCF routine of the current version of the NAG release has been used to reduce the Kompaneets equation into a system of ordinary differential equations (Dew \& Walsh 1981; Berzins et al. 1989; Berzins 1990; Skeel \& Berzins 1990). The D03PGF routine used in the first versions of KYPRIX is no longer available (see also Sect. 3.2). The two codes work adopting the same numerical framework or, in other words, the D03PCF routine of the current NAG release corresponds to the D03PGF routine of the NAG release used in the first versions of KYPRIX. On the other hand, they come from different technical implementations and exibit remarkable differences in several aspects. The main differences between the two routines, their use, and the corresponding implications for the code KYPRIX will be described in this work. To use the D03PCF routine, we have to put the Kompaneets equation in the form

$$
\sum_{j=1}^{\mathrm{NPDE}} P_{i, j} \frac{\partial U_{j}}{\partial Y}+Q_{i}=X^{-m} \frac{\partial}{\partial X}\left(X^{m} R_{i}\right)
$$

where $Y$ is the time variable and $X$ the spatial variable. Here $i=1$ and $\mathrm{NPDE}=1$, and $P_{i, j}, Q_{i}, R_{i}$ depend on $x, t, U, \partial U / \partial x$, the vector $U$ is defined below. In our case, $P_{i, j}=1$ and $m=0$ (Cartesian coordinates). Note that $P_{i, j}, Q_{i}, R_{i}$ do not depend on $\partial U / \partial t$.

The variables that enter in this equation are introduced and used in logarithmic form to have a good and essentially uniform accuracy of the solution in the whole frequency range under consideration. They are $X=\log (x)$ and $U=\log (\eta)$. Hereafter, we use the variables $X, U, Y$ for what concerns the informatic aspect of the problem, keeping the use of the variables $x, \eta, y$ 
for considerations directly linked to physical aspects. The function $R_{i}$ is determined only by the inverse Compton term, while the other physical processes, i.e. at least Compton scattering, bremsstrahlung, and radiative Compton, are included in the function $Q_{i}$. To reduce Eq. (7) into a system of ordinary differential equations, the D03PCF routine uses the method of lines: the right member of Eq. (7) is discretized, reducing the calculation of partial derivatives in terms of finite values of the solution vector $U$ on all the points of the $X$ axis grid. Spatial discretization is made by the method of finite differences (Mitchell \& Griffiths 1980). The resulting system of ordinary differential equations is solved using a differentiation formula method. The choice of the time parameter was driven by the need to have a very simple form of the Kompaneets equation. Finally, a "temperature independent" (time) Comptonization parameter

$Y=y(t)=\int \frac{\mathrm{d} y_{\mathrm{e}}}{\phi}=\int_{t_{i}}^{t} n_{\mathrm{e}} \sigma_{\mathrm{T}} c \frac{k T_{\mathrm{r}}}{m c^{2}} \mathrm{~d} t^{\prime}$,

has been found to be particularly advantageous (Burigana et al. 1991a).

\subsection{Boundary conditions}

Integrating equations of the type of Eq. (7) means to calculate the time evolution of the function $U(X, Y)$, for a given initial condition $U(X, 0)$. In fact, the problem is also called "problem at initial conditions". Numerically, the derivatives of $U$ are replaced by finite differences between values of $U$ computed on a grid of points (in $X$ ) and the differential equation is replaced by a system of more simple equations. However, in the presence of the only initial condition, this system is singular (Press et al. 1992). For this reason, resolving partial differential parabolic equations needs boundary conditions. In general, boundary conditions mean additional relations written to be joined to the system derived from the discretization to finite differences.

Therefore, a good statement of the problem needs the definition of appropriate boundary conditions. The capability of a refresh of these conditions along the integration in time leads more stability to the solution evolution because of the evolution of the radiation field. Thanks to the opportunity of having the correct value of $\phi$ for each time step, the update of the boundary conditions can be physically motivated (see also Sect. 3.2.6). The limits of the frequency range considered are $X_{\min }=\log \left(x_{\min }\right)=-4.3$ and $X_{\max }=\log \left(x_{\max }\right)=1.7$. Of course, we want a solution of the Kompaneets equation over all the frequency range where it is possible to measure the CMB. Also, we need to consider a frequency range wide enough to contain, in practice, all the energy density of the cosmic radiation field. The frequency range is so wide for the other two reasons described below.

During the time evolution, some spurious oscillations of the solution may appear at points close to the boundaries. These effects may be partially amplified if, for the computational reasons discussed in the following, the necessary refreshing of the electronic temperature is not made for every time step (but they could also occur independently of the need to refresh $\phi$ - for example for cases at constant $\phi$ ). Fixing the frequency integration range limits far from the interval where we are interested in computing the photon distribution function allows prevention of the "contamination" of these solution by possible spurious oscillations in the frequency range of interest.

We can generally assume that a Planckian spectrum at $x_{\min }$ is formed before recombination on a shorter timescale than the expansion time and, in contrast, at $x_{\max }$ the shape of the spectrum is unknown. Thus, we implemented in the code the possibility of adopting a particular case of Neumann boundary conditions, i.e. the requirement that the current density, in the frequency space, is null at the boundaries of the integration range (Chang \& Cooper 1970):

$\left[\phi \frac{\partial \eta}{\partial x}+\eta(1+\eta)\right]_{x=x_{\min }, x_{\max }}=0$.

This choice of boundary conditions formally satisfies the requirement of the problem when we integrate the Kompaneets equation in the case of Bose-Einstein-like distortions (with a frequency dependent chemical potential, $\mu=\mu(x)$, vanishing at very low frequencies). In fact, such distorted spectra are indistinguishable from a blackbody spectrum at sufficiently high and at low frequencies.

Of course, it is possible to make a different choice of the boundary conditions by selecting Dirichlet-like conditions. In this case the photon occupation number at the boundaries of the integration interval does not change for the whole integration time. (In general cases, keeping constant conditions at the boundaries could be dangerous for the continuity of the solution. Nevertheless, this condition can work for some specific problems - typically for problems with constant $\phi$ ).

\section{A detailed view on KYPRIX}

The code KYPRIX was written to solve the Kompaneets equation in many kinds of situations. The physical processes that can be considered in KYPRIX are: Compton scattering, bremsstrahlung, radiative Compton scattering, sources of photons, energy injections without photon production, energy exchanges (heating or cooling processes) associated to $\phi \neq 1$ at low redshifts, radiative decays of massive particles, and so on (see e.g. Danese \& Burigana 1994 for some applications). This code could be easily implemented to consider other kinds of physical processes. Various kinds of initial conditions for the problem can be considered, and many of them have already been implemented in KYPRIX. The first obvious case is a pure Planckian spectrum. Several ways to model an instantaneuos heating implying deviations from the Planckian spectrum have been introduced: a pure Bose-Einstein (BE) spectrum or a BE spectrum modified to become Planckian at low frequencies (this option could be exploited to integrate the Kompaneets equation with a constant $\phi$ and constant boundary conditions); a grey-body spectrum; a superposition of blackbodies.

The data are saved in five files:

DATI. This file contains the information about the specific parameters of the problem with a general description of its main aspects.

DATIP. In this file we give the evolution of interesting quantities, such time, redshift, $\phi$, and other quantities inherent to the physical and numerical aspects of the problem (see also Sect. 5.1).

DATIG. It contains the grid of points for the $X$ axis used by the main program (remember that we are using a dimensionless frequency), a Planckian spectrum at temperature $T_{0}$, and the solution vector $\boldsymbol{U}$ (that is to say $\log (\eta))$ at $y=0$ (starting time).

DATIDE. This is the fundamental output file, which gives the solution of the Kompaneets equations at the desired cosmic epochs. 
DATIT It is similar to the file DATIDE, but it contains the solution in term of brightness temperature (i.e. equivalent thermodynamic temperature; see Eq. (12) in Sect. 3.2.2).

\subsection{Main subdivisions}

The code is divided into several sections and, from a general point of view, is structured as described here.

1. the main program, where many actions can be carried out: choice of the physical processes, choice of the cosmological parameters, initial conditions, characteristics of the numerical integration (accuracy, number of points of the grid), time interval of interest, choice of the boundary conditions, chemical abundances, ionization history;

2. subroutine PDEDEF is the subprogram where the problem is numerically defined. This subroutine is also divided in subsections to allow modifications in a simple and practical way;

3. subroutine BNDARY. Here the boundary conditions are numerically specified;

4. Subroutines and auxiliary functions perform specific calculations.

\subsection{Technical specifications and code implementation}

The first version ${ }^{3}$ of KYPRIX worked with the Mark 8 version of the NAG numerical library and were based on the routine D03PGF. The version of the NAG numerical library currently distributed is the Mark 21, so an update of the code KYPRIX is necessary to adapt it to this new package.

When KYPRIX starts running, it asks for all the input data: from the declarations of the output files' names to the integration accuracy and features. In the following sections we give a description of the various aspects of the code (and of its update), trying to give relevant hints about computational aspects of the code.

\subsubsection{Grid}

The frequency integration interval is divided into a grid of points (the mesh points): the larger the number of points, the smaller the adopted frequency step. We adopted an equispaced grid in $X$. It is possible to use a very dense grid (for example 36001 mesh points corresponding to 36000 frequency steps). In general, it is necessary to use at least 3001 mesh points to have an accurate enough solution.

We find a big difference between the two NAG versions, not reported in the documentation of the routine D03PCF. In the first version (D03PGF), the subroutine where the partial differential equation is defined adopted the same mesh points defined in the main program. In the Mark 21 version the calculation is carried out in a different manner: the mesh points used in the subroutine PDEDEF is shifted of half spatial step with respect to the mesh defined in the main program. In this way, the mesh points in the PDEDEF subroutine will be exactly in the middle of the steps defined in the grid of the main program. For this reason, the limits of the integration interval are not considered in the mesh points in the subroutine PDEDEF, and they are used only for the boundary conditions.

The effect of this feature implies the definition of new parameters that play a fundamental role in the subroutine PDEDEF.

\footnotetext{
3 Written in 1989 by C. Burigana.
}

The integral quantities in the Kompaneets equation (necessary to define the radiative Compton term in the kinetic equations and the electron temperature) are computed once for any time step, inside the PDEDEF subroutine. For this computation, arrays of dimension equal to the number of mesh points of the $x$ variable as defined in the PDEDEF subroutine are used. Therefore, a particular care must be taken in the definition of the dimension of the arrays defined in KYPRIX. Those used in the main program have dimensions equal to the number of points of the mesh defined in the main program. The same dimension is given for the arrays defined for the boundary conditions. On the other hand, the major number of arrays are used in the PDEDEF subroutine to compute the integral quantities. The "inner" grid adopted in the PDEDEF subroutine is based on mesh points in the middle of the spatial steps of the main program grid, so the two grids can not work with the same point number; in fact, the arrays used in the PDEDEF subroutine have dimension NPTS - 1 . Therefore, in the main program and in the subroutine BNDARY we have to work with arrays based on the formula:

$X(I)=A+(I-1) \times \frac{(B-A)}{(\mathrm{NPTS}-1)}$,

with $1 \leq X \leq$ NPTS,

to define the correspondence between the grid of NPTS points and the $X$ position, while we need another expression able to shift the grid in the PDEDEF subroutine by a half step and based on NPTS - 1 mesh points:

$X(I)=\left(A+(I-1) \times \frac{(B-A)}{(\mathrm{NPTS}-1)}\right)+\left(\frac{(B-A)}{2(\mathrm{NPTS}-1)}\right)$,

with $1 \leq X \leq$ NPTS -1

For continuity reasons, we need to define (according to the choices made in the main program) the solution vector, containing the photon initial distribution function, at the beginning of the integration also according to this grid definition. This vector is used by the PDEDEF subroutine as the initial spectrum adopted for the computation of the rates of the physical processes, and, of course, it is then renewed at every time step incrementation.

\subsubsection{Output}

Concerning the output files, the updated version of KYPRIX stores a new vector containing the "inner" $X$ grid used by the PDEDEF subroutine, XXGR (XGR refers to the main program $X$ grid).

In addition, we preferred to have the possibility of performing the conversion of the solution into equivalent thermodynamic temperatures directly into the code and save it in a new output file (DATIT). The conversion relation is

$T_{\text {term,equiv }}=\frac{x T_{0}}{\ln (1+1 / \eta)}$

(we remember that in the code $X=\log _{10}(x)$ and $U=\log _{10}(\eta)$ ).

The fundamental reason for performing this conversion directly in the code is associated to the extreme accuracy required for the solution in the case of very small distortions, of particular interest given the FIRAS results (Fixsen et al. 1996). During the first tests, the conversion of the solution in brightness temperature was performed at the same time as the solution visualization, through the IDL visualization program. Saving the solution 
into files is typically performed, not for all the points of the grid, but for a reduced grid of, for example, 300 equidistant points along the original grid to avoid to store files of large size, useless for our scope, given the interest for the CMB continuous spectrum (by definition, the Kompaneets equation is not appropriate to treating recombination lines). If the considered distortions were very small, then the solution at each specific "inner" grid point could be affected by a numerical uncertainty that is not negligible in comparison with the very small deviations from a Planckian spectrum relevant in these cases. This numerical error is greatly reduced (becoming negligible for our purposes) by the averaging over a suitable number of grid points. Of course, the storing of the solution directly on a limited number of grid points makes this averaging no longer possible on the stored data. It is then necessary to average the solution values in intervals corresponding to the output $x$ grid directly into the code. In many circumstances, the diagram shape derived by applying the conversion to brightness temperature only on the stored averaged solution still deviates at high frequencies from the effectively computed solution displayed by considering all the "inner" grid points because of the high gradients in the photon distribution function and/or in the brightness temperature that makes it difficult, or impossible, to find a general rule for the solution binning that simultaneously works properly for the two solution representations. This problem is avoided by converting the solution vector in equivalent thermodynamic temperature before the binning of its values and then applying the binning to the equivalent thermodynamic temperature. The result is then a very clean and precise brightness temperature diagram, even for very small distortions.

Other minor changes we made in the output data, where we pass from real to double precision, and in the frequency of storing results into the output files.

\subsubsection{Equation formalism}

A necessary update of the code was performed to adapt it to the different formalism adopted by the new version of the NAG routine. This regards the expression of the Kompaneets equation in the PDEDEF subroutine. In particular, the D03PGF routine adopted the following expression of the partial differential equation:

$C_{i} \frac{\partial U_{i}}{\partial Y}=X^{-m} \sum_{j=1}^{\mathrm{NPDE}} \frac{\partial}{\partial X}\left[X^{m} G_{i j} \frac{\partial U_{j}}{\partial X}\right]+F_{i}$,

where $i=1,2, \ldots$, NPDE (number of partial differential equations); $C_{i}, F_{i}$ depends on $X, Y, U, \partial U / \partial X ; G_{i, j}$ depends on $X, Y, U$ and $U$ is the set of solutions values $\left(U_{1}, U_{2}, \ldots, U_{\mathrm{NPDE}}\right)$. The expression now adopted by the D03PCF routine is represented by Eq. (7).

It is simple to translate the code from the old to the new formalism. In the case NPDE $=1$. In this case, we have simply that $R_{1}$ contains both the function $G_{1}$ and the vector solution derivative with respect to $X$ according to

$R_{1}=G_{11} \times \frac{\partial U_{1}}{\partial X}$.

At this point, it is necessary to apply only the following substitutions:

$Q_{1}=-F_{1} \quad$ and $\quad P_{11}=C_{1}$.
With respect to this formalism, it is not difficult to adapt the various terms of the Kompaneets equation to the D03PCF routine. The terms that describe radiative Compton, bremsstrahlung, (optional) electromagnetic processes, and part of the contribution of the Compton scattering are counted in the function $Q_{1}$. Instead, the second derivative of the solution vector with respect to $X$, which represents part of the inverse Compton rate, is counted in the function $R_{1}$, so, according to these settings, we can write:

$Q_{1}=\mathrm{FC}+\mathrm{FBREM}+\mathrm{FRAD}+\mathrm{FDEC}$,

where FC stands for the contribution of the Compton scattering, FBREM the bremsstrahlung one, FRAD the radiative Compton one, and FDEC represents the contribution of the radiative decaying of particles. These terms are written in the form

$$
\begin{aligned}
\mathrm{FC}= & {\left[\phi \frac{\partial U}{\partial X}\left(\frac{\partial U}{\partial X}+3\right)+10^{X}\left(\frac{\partial U}{\partial X}+4\right)\right.} \\
& \left.+2 \times 10^{X} 10^{U}\left(\frac{\partial U}{\partial X}+2\right)\right] \frac{1}{\ln (10)}
\end{aligned}
$$

and

$$
\begin{aligned}
& \text { FBREM + FRAD }=\frac{\left(\mathrm{e}^{10^{X}}\right)^{\phi}}{10^{3 X}} \times\left\{\frac{1}{10^{U}}-\left[\left(\mathrm{e}^{10^{X}}\right)^{\phi^{-1}}-1\right]\right\} \\
& \times\left(\mathrm{FF} 0 \times W \times 1.5 \phi^{-1 / 5} \times \mathrm{FGAUNT}+\frac{\mathrm{DC} 0}{W} I_{1} \times \mathrm{GDC}\right),
\end{aligned}
$$

where FF0 and DC0 are the coefficients for the rates of bremsstrahlung and radiative Compton, respectively; FGAUNT and GDC represent the Gaunt factor corrections for bremsstrahlung and radiative Compton, respectively; $I_{1}$ is an integral quantity refreshed at every time step (see also Sect. 3.2.7).

The inverse Compton contributes to $R_{1}$ in this way:

$R_{1}=\frac{\partial U}{\partial X} \times \phi \times \ln (10)^{-2}$

Finally, we can put $P_{11}=1$.

Furthermore, it is possible to add other source terms ${ }^{4}$ in Eq. (16).

\subsubsection{Boundary conditions}

Also notable are the differences between the input expressions defining the boundary conditions. The D03PGF routine adopted an expression of the form

$P_{i}(Y) U_{i}+Q_{i}(Y) \frac{\partial U_{i}}{\partial X}=R_{i}(Y, U)$

where $i=1,2, \ldots$, NPDE and $P_{i}(Y), R_{i}(Y, U), Q_{i}(Y)$ are functions to be defined. A quite different notation is used to provide the boundary conditions in the D03PCF routine

$\beta_{i}(X, Y) R_{i}\left(X, Y, U, U_{X}\right)=\gamma_{i}\left(X, Y, U, U_{X}\right)$,

where $i=1,2, \ldots, \mathrm{NPDE}$ and $\beta_{i}(X, Y) R_{i}\left(X, Y, U, U_{X}\right)$ and $\gamma_{i}\left(X, Y, U, U_{X}\right)$ are functions to be defined $\left(U_{X} \equiv \partial U / \partial X\right)$. As

\footnotetext{
${ }^{4}$ For example, an already implemented subroutine models a term, called FDEC here, to be added in Eq. (16), which acconts for contributions of possible radiative decays of massive particles in the primordial
} Universe. 
a consequence of this notation, Neumann like boundary conditions can be now specified according to the expression

$\beta(1)=1$

$\gamma(1)=-X V A \times\left(10^{U(1)}+1\right) \times \ln 10^{-2}$,

where $X V A$ is the vector related to the $X$ position computed in $\mathrm{A}$, and the dimension of both the equations corresponds to the differential equation number. Similar conditions are defined for the other extreme of the integration interval $[A, B]$.

\subsubsection{Accuracy parameters}

Another considerable difference between the two library versions regards the definition of the integration accuracy parameter. D03PGF used three parameters for monitoring the local error estimate in the time direction, supplying a good versatility. RELERR and ABSERR were respectively the quantity for the relative and absolute components to be used in the error test. The third parameter, INORM, was used to define the error test. If $E(i, j)$ is the estimated error for $U_{i}$ (the vector solution) at the $j$ th point of the $X$ grid, then the error test was

$$
\begin{aligned}
& \text { - } \operatorname{INORM}=0 \Rightarrow|E(i, j)| \leq \operatorname{ABSERR}+\operatorname{RELERR} \times|U(i, j)| \\
& \text { - } \operatorname{INORM}=1 \Rightarrow|E(i, j)| \leq \operatorname{ABSERR}+\operatorname{RELERR} \times \\
& \quad \max _{y}|U(i, j)| \\
& \text { - } \operatorname{INORM}=2 \Rightarrow\|E(i, j)\| \leq \operatorname{ABSERR}+\operatorname{RELERR} \times\|U(i, j)\| .
\end{aligned}
$$

Instead, according to the new library version we have to define only one parameter ACC, a positive quantity that monitors the local error in the time integration. If $E(i, j)$ is defined as above, then the error test is

$|E(i, j)|=\operatorname{ACC} \times(1+|U(i, j)|)$.

This is equivalent to the error test implemented in the D03PGF routine in the case INORM $=0$ and ABSERR $=$ $\operatorname{RELERR}(=\mathrm{ACC})$.

\subsubsection{Electron temperature}

During the numerical integration, some subprograms use the distribution function calculated at that time to compute $\phi$. The integrals to be computed are those that we find in the expression for $\phi_{\mathrm{eq}, \mathrm{C}}$ :

$\phi_{\mathrm{eq}, \mathrm{C}}=\frac{T_{\mathrm{e}}}{T_{\gamma}}=\frac{\int_{0}^{\infty} \eta(\eta+1) x^{4} \mathrm{~d} x}{4 \int_{0}^{\infty} \eta x^{3} \mathrm{~d} x}$.

In this calculation, the integration range is obviously the integration interval considered for the problem: $A \leq X \leq B$ (that, in terms of mesh ordering, corresponds to the range between 1 and NPTS or NPTS - 1). All the points of the grid are used to compute these integrals. The integration is based on the NAG D01GAF routine, suitable for tabulated functions. The update value of $\phi$ is also used in the boundary conditions.

In the previous version of the code KYPRIX, integral quantities were computed through a specific modification of the NAG package implemented by the KYPRIX code author that allowed recovery of the whole vector solution at each time step in the subroutines (and in particular in PDEDEF), while the original package made the solution only available in PDEDEF separately at each grid point (the package originally designed for "pure" partial differential equation, without terms involving integrals of the solution). This modification, possible thanks to the availability of the NAG sources (and, in practice, thanks to the relative simplicity of the early library versions), permitted updating the integral quantities perfectly according to the "implicit" scheme adopted by the code for the integration in time. This is no longer feasible. Therefore, the update of the integral quantities must now be performed with a "backward" scheme, saving the solution at the previous time step in a proper vector and using it in the computation at the given time step. As is well known, "backward" schemes are typically less stable than implicit schemes. And, in fact, we verified in some cases the difficulty of the D03PCF routine to work implementing the update of the quantities corresponding to the integral terms in the Kompaneets equation (and in particular of $\phi$ ) for each time step. This was likely caused by numerical instabilities.

We then introduced a new integer control parameter into the code: STEPFI. It determines the frequency for the update of the dimensionless electron temperature $\phi$, relevant, of course, in case we want to perform an integration with a variable $\phi$. We have checked that updating the integral terms in the Kompaneets equation not at every time step, but after a suitable number of time steps does not affect the accuracy of the solution. This is because the time increasing in the code is performed with very small steps, while the physical variation of $\phi$ occurs on longer timescales ${ }^{5}$. See Sect. 5.2.2 for tests regarding the implications of this new implementation of the electron temperature evolution.

\subsubsection{Radiative Compton}

In computing of the radiative Compton term there is an integral term, $I_{1}$, given by the numerator of the right member of Eq. (25) (see Eq. (16) in Burigana et al. 1995), so it is necessary to harmonize its update according to the parameter STEPFI discussed in the previous subsection. In fact, a possible asynchronous update of it and $\phi$ could create numerical instabilities and the crash of the code run, as physically evident from the strong relevance of both radiative Compton term (at least at high redshifts) and electron temperature for the evolution of the low-frequency region of the spectrum.

\subsubsection{Integration routines}

The global accuracy of the code KYPRIX depends on the accuracy of the solver for the partial differential equation, as well as on the accuracy of all the other routines dedicated to different specific computations. In this section we focus on the routines of numerical integration used in the KYPRIX. As discussed in the previous sections, the D01GAF routine has been used for tabulated functions. On the other hand, KYPRIX involves computing the integrals of various functions defined by analytical expressions, namely the parameters characterizing different types of initial conditions for the distorted spectra, such as the amount of fractional injected energy, the electron temperature, and the relationship between the different time variables entering into the code (see also Sect. 4.1). Ultimately, the better accuracy in these specific computations implies a better global accuray for KYPRIX. In the early release of the code KYPRIX, the NAG D01BDF routine was used to calculate integrals of a

\footnotetext{
5 Of course, for physical processes with a stronger variation in the electron temperature, the accuracy parameter (see previous subsection) should be good enough to force the code to adopt sufficiently small time steps.
} 
function over a finite interval. The same task can be carried out by the D01AJF routine. This code offers a better accuracy than D01BDF (D01AJF is in fact suitable also to integrating functions with singularities, both algebraic and logarithmic). After the routines substitution, the results show a strong increase in accuracy. In particular, this improvement offers the possibility of also investigating very small distortions that require a very precise determination of all the relevant quantities because the absolute numerical error of the integration must be much smaller than the (very small quantities) of interest in these cases. In particular, the quantity $\Delta \epsilon_{r} / \epsilon_{i}$ (where $\epsilon_{r}$ is the actual density energy and $\epsilon_{i}$ the energy density corresponding to the unperturbed distribution function just before the energy injection) must be constant during all the integration process in the absence of energy injection terms, according to the energy conservation. The increase in precision on the computation of this quantity was noteworthy, now always keeping inside a few percent of the physical value (and its possible physical variation; see Sect. 5.1) of the same quantity independently of the magnitude of the considered distortion, at the same time allowing an accurate check of the global accuracy of the code, improved thanks to better computation of all the integral terms appearing in the Kompaneets equation. The remarkable improvement in energy conservation, even in the case of very small distortions, is an important feature of the new version of KYPRIX that makes is applicable to a wider set of cases.

\section{New physical options}

\subsection{Cosmological constant}

About ten years ago, the relevance of the cosmological constant term (or of dark energy contribution) was probed by a wide set of astronomical observations of type Ia supernovae (Perlmutter et al. 1999; Riess et al. 1998). We then updated the numerical integration code KYPRIX to include the cosmological constant in the terms controlling the general expansion of the Universe. In particular the input background cosmological parameters considered in the code are now $T_{0}, \kappa, h\left[=H_{0} /\left(100 \mathrm{~km} \mathrm{~s}^{-1} \mathrm{Mpc}^{-1}\right)\right], \Omega_{\mathrm{nr}}, \Omega_{\mathrm{b}}, \Omega_{\Lambda}, \Omega_{\mathrm{K}}$, i.e. the present $\mathrm{CMB}$ temperature, the contribution of massless neutrinos, the Hubble constant, the (non relativistic) matter and baryon energy density, the energy densities corresponding to cosmological constant and curvature terms.

To compute the proper cosmic evolution of the various terms, a scale factor parameter $\omega$ (increasing with time) (Silk \& Stebbins 1983), defined by

$\omega=\frac{a}{a_{1}} \equiv \frac{m_{\mathrm{e}} c^{2}}{k T_{0}} \frac{1}{1+z}=1.98 \times 10^{9} \Theta(1+z)^{-1}$,

has been adopted (here $\Theta \equiv T_{0} / 3^{\circ} \mathrm{K}$, and the index 1 refers to a particular epoch, when the CMB energy density was equal to the electron mass $\left.{ }^{6}: k_{\mathrm{T}}\left(a_{1}\right)=m_{\mathrm{e}} c^{2}\right)$. To write a suitable expression for its time evolution we have to introduce two new key parameters

$\beta=\frac{\rho_{m 1}}{\rho_{r 1}}=3.5 \times 10^{-6} \frac{h^{2}}{\Theta^{3}} \Omega_{\mathrm{tot}}$,

which is the initial ratio between matter energy density and radiation energy density, and

$\frac{1}{\tau_{g 1}}=\left(\frac{8 \pi}{3} G \rho_{r 1}\right)^{1 / 2}=\left[\frac{8 \pi}{3} G \frac{a}{c^{2}}\left(\frac{m_{\mathrm{e}} c^{2}}{k}\right)^{4}\right]^{1 / 2}=0.076 \mathrm{~s}^{-1}$

6 The parameter $\omega$ is analogous to the scale factor $a$, but normalized at the epoch in which $a=a_{1}$, that is to say when $k T=m_{\mathrm{e}} c^{2}$. defined as a initial gravitational time scale. The quantities with the index 1 refer to the epoch when $a=a_{1}$, with the index 0 when $t=t_{0}$ (today); $\rho_{r 1}$ and $\rho_{m 1}$ are related to $\rho_{\mathrm{r}}$ and $\rho_{m}$ by

$\rho_{\mathrm{r}}=\rho_{0 r}\left(\frac{\omega_{o}}{\omega}\right)^{4}=\rho_{r 1} \frac{1}{\omega^{4}} ; \quad \rho_{m}=\rho_{0 m}\left(\frac{\omega_{0}}{\omega}\right)^{3}=\rho_{m 1} \frac{1}{\omega^{3}}$,

respectively.

Now we can define an equation for the evolution of $\omega$ :

$$
\begin{aligned}
\frac{\dot{\omega}}{\omega} & =\left[\frac{8 \pi}{3} G \rho(\omega)\right]^{1 / 2} \\
& =\frac{8 \pi}{3} G\left[\frac{\rho_{r 1} \kappa}{\omega^{4}}+\frac{\rho_{m 1}}{\omega^{3}}+\frac{\rho_{K 1}}{\omega^{2}}+\rho_{\Lambda}\right],
\end{aligned}
$$

where we have included the contribution of massless relativistic neutrinos in the term $\kappa$ (see also footnote 1 . The term $\kappa$ should be properly evaluated considering also possible energy injections after neutrino decoupling). After some calculations, we can finally write the completed and updated expression of $\mathrm{d} t / \mathrm{d} \omega$ :

$\frac{1}{\dot{\omega}}=\frac{\tau_{g 1} \omega}{\left[1+\beta \omega\left(1+\frac{\Omega_{K / m} \omega}{2.164 \times 10^{9}}+\frac{\Omega_{\Lambda / m} \omega^{3}}{2.164 \times 10^{27}}\right)\right]^{1 / 2}}$,

where $\Omega_{x / y}=\Omega_{x} / \Omega_{y}$.

The equation for $\dot{\omega}$ has to be inserted in the expression giving $\mathrm{d} y=a_{\mathrm{c}} \mathrm{d} t$, which is inside the integral used to compute the time variable $y(\omega)=\int_{\omega_{\text {start }}}^{\omega} \mathrm{d} y$ because we set $y=0$ when the integration starts at $\omega=\omega_{\text {start }}$ (or equivalently at $z=z_{\text {start }}$ ). Finally, the expression for the time evolution of $\omega$ and $y$ are related by the variable change:

$\mathrm{d} y=a_{\mathrm{c}} \mathrm{d} t=a_{\mathrm{c}} \frac{\mathrm{d} t}{\mathrm{~d} \omega} \mathrm{d} \omega=a_{\mathrm{c}} \frac{\omega}{\dot{\omega}} \frac{1}{\omega} \mathrm{d} \omega$,

where $a_{\mathrm{c}}=\phi /\left(\tau_{\mathrm{c} 1} \omega^{4}\right)\left(\tau_{\mathrm{c} 1}=2.638 \times 10^{-9} \Theta^{3} /\left(h^{2} \Omega_{\mathrm{b}}\right)\right)$.

Implementation of the cosmological constant and curvature terms makes the code KYPRIX suitable to being applied to interesting cases at late ages, including cosmic epochs at $z<1$ when $\Lambda$ supplies the greatest contribution to the expansion rate of the Universe. Remarkable examples are spectral distortions associated to the re-ionization of the Universe, which starts at relatively low redshifts $(z \lesssim 10-20)$ in typical astrophysical scenarios. For the sake of completeness, a few words are needed about the computation of the time evolution in the code. The subroutine called WDIY0 is the core of the time evolution in KYPRIX: it computes the value of $\omega$ given a value of $y$. This process takes advantage of the definition of $y$ as the integral of $\mathrm{d} y$ and, of course, it happens at each time step. To do this, we make use of a double precision version of the function ZBRENT, from "Numerical Recipes" (Press et al. 1992).

\subsection{Chemical abundances}

In this new version of the code, it is possible to choose the primordial abundances of $\mathrm{H}$ and $\mathrm{He}$. We have consistently computed the electron number density, $n_{\mathrm{e}}$, involved in the different physical processes. In the previous implementation, it was assumed a fixed abundance of $\mathrm{H}$ and $\mathrm{He}$ (and full ionization). The electron number density was then given by

$n_{\mathrm{e}}^{\text {free }}=n_{\mathrm{e}}^{\text {tot }} \simeq \frac{\rho_{\mathrm{b}}}{m_{\mathrm{b}}} \frac{7}{8}$, 
where $\rho_{\mathrm{b}}$ is the baryon density and $m_{\mathrm{b}}$ the mean mass of a baryon. Now, denoting by $f_{\mathrm{H}}$ the fraction in mass of primordial $\mathrm{H}$ and considering that $n_{\mathrm{e}}=n_{\mathrm{H}}+2 n_{\mathrm{He}}$, we have

$n_{\mathrm{e}}^{\text {free }}=n_{\mathrm{e}}^{\text {tot }}=\frac{1+f_{\mathrm{H}}}{2} \frac{\rho_{\mathrm{b}}}{m_{\mathrm{b}}}$.

This obviously affects the physical processes involved in the code. Compton scattering, radiative Compton, and bremsstrahlung depend linearly ${ }^{7}$ on $n_{\mathrm{e}}^{\text {free }}$.

\subsection{Ionization history}

In the past years, CMB observations are achieving a very high accuracy, in particular regarding the angular power spectrum of temperature anisotropies, but also for that of E mode polarization and cross-correlation (see e.g. Nolta et al. 2009). A detailed understanding of the cosmological reionization process is crucial for precisely modeling the power spectrum of $\mathrm{CMB}$ anisotropies in comparison with current data, while a better treatment of recombination is very relevant in view of the data expected by the forthcoming ESA Planck satellite ${ }^{8}$ (The Planck Collaboration 2006).

Accurate modeling of the ionization history is also crucial for precisely computing of CMB spectral distortions in view of the comparison with data from a future generation of highsensitivity experiments. We then included these aspects in the current implementation of KYPRIX. In particular, the fraction of each state of ionization of the relevant elements ( $\mathrm{H}$ and $\mathrm{He}$ ) has been implemented.

The effects of this implementation is negligible ${ }^{9}$ in practice for the radiative Compton, because this process is important at high redshifts when the medium is fully ionized. Instead, Compton scattering and bremsstrahlung rates are significantly influenced by this implementation. Once introduced, the electron ionization fraction in the code, $\chi_{\mathrm{e}}$, which gives the number of electrons that takes part in the physical processes, we can choose different ways the active fractions of elements can play their roles in the phenomena.

Given $\chi_{\mathrm{e}}$, from the charge conservation law, we have a constraint on the number of the free ions in the considered plasma. The simplest way to account for them in the code is to assume an equal fraction of ionization for $\mathrm{H}$ and $\mathrm{He}$. Of course, this is a toy model, but this parametrization was very useful for testing the code.

A somewhat more accurate treatment of the physics of reionization/recombination processes implemented in the code is based on the Saha equation:

$\frac{n_{i+1} n_{\mathrm{e}}}{n_{i}}=\frac{2}{\Lambda^{3}} \frac{g_{i+1}}{g_{i}} \mathrm{e}^{-\frac{\epsilon_{i+1}-\epsilon_{i}}{k_{\mathrm{B}} T}}$,

where $n_{i}$ is the density of atoms in the $i$ th state of ionization, $n_{\mathrm{e}}$ the electron density, $g_{i}$ the degeneracy of states for the $i$ ions, $\epsilon_{i}$ the energy required to remove $i$ electrons from a neutral

\footnotetext{
${ }^{7}$ For bremsstrahlung, the dependence on the densities of nuclei is now explicit.

${ }^{8}$ http://www.rssd.esa.int/planck

${ }^{9}$ For example, for $z \lesssim 10^{-4}$ and $\Delta \epsilon_{r} / \epsilon_{i}=10^{-5}$ we find that, for both $\mathrm{BE}$ and Comptonization like distortions, the rate of radiative Compton is less than $1 / 1000$ of the rate of bremsstrahlung at each frequency of the grid.
}

atom, and $\Lambda$ the thermal de Broglie wavelength of an electron, defined by

$$
\Lambda=\sqrt{\frac{h^{2}}{2 \pi m_{\mathrm{e}} k_{\mathrm{B}} T}} .
$$

Providing the electron ionization fraction defined as ${ }^{10}$

$\chi_{\mathrm{e}} \equiv \frac{n_{\mathrm{e}}^{\text {free }}}{n_{\mathrm{e}}^{\text {tot }}}$,

we can compute the unknowns $\chi_{\mathrm{H}}, \chi_{\mathrm{H}^{+}}, \chi_{\mathrm{He}}, \chi_{\mathrm{He}^{+}}, \chi_{\mathrm{He}^{++}}$, defined as the relative abundances of the different ionization states of each element with respect to the global number density of the element. The Saha equation provides the ratio between two state of ionization of a single specie, once given the electron density and the temperature:

$\frac{n_{\mathrm{H}^{+}}}{n_{\mathrm{H}}}, \frac{n_{\mathrm{He}^{+}}}{n_{\mathrm{He}}}, \frac{n_{\mathrm{He}^{++}}}{n_{\mathrm{He}^{+}}}$

To recover all the unknowns we need other relations. These additional conditions are provided by the charge conservation and the nuclei conservation. From charge conservation it is possible to recover the contribution of the electrons related to a single specie to the total number of free electrons. The latter is of course related to the ionization fraction of all the involved species and can be written as:

$n_{\mathrm{e}}^{\text {free }}=\frac{\rho_{\mathrm{b}}}{m_{\mathrm{b}}}\left[\chi_{\mathrm{H}^{+}} f_{\mathrm{H}}+2\left(\frac{1-f_{\mathrm{H}}}{4}\right)\left(\chi_{\mathrm{He}^{+}}+\chi_{\mathrm{He}^{++}}\right)\right]$,

where $f_{\mathrm{H}}$ is the fraction of primordial $\mathrm{H}$ described in the previous section. In Eq. (39) it is possible to identify the number of electrons coming from $\mathrm{H}$ and from $\mathrm{He}$.

The nuclei conservation law separately for $\mathrm{H}$ and $\mathrm{He}$ is expressed by

$n_{\mathrm{H}}^{\mathrm{tot}}=\frac{\rho_{\mathrm{b}}}{m_{\mathrm{b}}}\left[f_{\mathrm{H}}\left(\chi_{\mathrm{H}}+\chi_{\mathrm{H}^{+}}\right)\right]$

$n_{\mathrm{He}}^{\mathrm{tot}}=\frac{\rho_{\mathrm{b}}}{m_{\mathrm{b}}}\left[\left(\frac{1-f_{\mathrm{H}}}{4}\right)\left(\chi_{\mathrm{He}}+\chi_{\mathrm{He}^{+}}+\chi_{\mathrm{He}^{++}}\right)\right]$.

Providing the electron ionization fraction and the temperature, it is possible to build two separate systems (one for $\mathrm{H}$ and one for $\mathrm{He}$ ) in order to recover the considered ionization fractions. The code can ingest a table with the desired evolution of $\chi_{\mathrm{e}}$ and, as needed for a physical modeling of cosmological reionization, of the electron temperature.

The best way to perform the exact calculation of the rates of considered processes in scenarios involving reionization/recombination uses a co-running code, coupled to KYPRIX, able to supply the ionization fraction for all the species. For the recombination process, we developed an interface that allows the code to call an external program, in our case RECFAST (Seager et al. 1999), and run it with the same cosmological parameters selected for KYPRIX. Then KYPRIX will read the output table from RECFAST and use the ionization fractions for the calculation of the rates of the processes, allowing a more detailed estimation of the spectral distortion arising during the recombination process (Wong et al. 2008). If we are interested in standard recombination, we adopt the equilibrium electron temperature (Eq. (25)). A consistent modeling of a modified recombination should provide the evolution of both electron temperature and ionization fractions (at least $\chi_{\mathrm{e}}$ ).

${ }_{10}$ It is common to find $\chi_{\mathrm{e}}$ normalized to the $\mathrm{H}$ number density in the
literature. Obviously, the code can switch between the two conventions. 


\section{Tests on code performance, reliability, and accuracy}

Once terminated the updating of the numeric integration code, we carried out many accuracy and performance tests. Obviously, we checked the physical meaning of the numerical solutions provided by the code comparing them with the existing analytical solutions that in some cases can be considered as good approximations of exact solutions. In general, a code of good quality must have high numerical precision compared to the knowledge, both theoretical and observational, of the problem. Various routines, mainly from the NAG package, but also from the "Numerical Recipes" (Press et al. 1992) package, we used for several specific computations. Since different routines can solve the same mathematical problems using different numerical methods and/or implementations with different settings and input parameters, we verified that the adopted routines allow the appropriate accuracy and efficiency. In the following we report on some specific quality tests of the numerical solutions. We comment now briefly on the code computational time.

To evaluate the global CPU time of the code, we performed many runs with very different settings. These times we carried out testing the code on a machine with 4 Alpha CPUs, but effectively using only one CPU (now we are running the code on IBM Power5 Processors). The code's global CPU time ranges from a few minutes, for cases in which the integration starts at low redshifts, to about $5 \mathrm{~h}$ for some cases starting at very high redshifts $(y(z) \simeq 5)$. There are many variables that play a role in determining the global CPU time. The complete Kompaneets equation in fact involves several terms. In the code KYPRIX we can select the physical processes to be considered in the numerical integration, and the global CPU time increases with the number of activated processes.

Of course, the global CPU time depends on the parameters related to the numerical integration characteristics. The number of points adopted for the $X$ grid (see Sect. 3.2.1) has a strong influence on the global CPU time. Clearly, the integration accuracy improves with NPTS, and in many cases it must be set to a very high value. We find that the global CPU time is approximately proportional to NPTS $\left(t_{\mathrm{CPU}} \propto \mathrm{NPTS}\right)$.

The parameter that plays the most relevant role in determining the global CPU time is the accuracy required for the time integration. The final solution precision depends on the value of the corresponding parameter ACC. Only for very high accuracy (ACC $\lesssim 10^{-12}-10^{-14}$ ) does the CPU time reach the duration of some hours while keeping ACC $\sim 10^{-5}$ the integration is carried out in a few minutes. The limits imposed by CMB spectrum observations drive us to investigate the small distortions in particular. It is then necessary to work with low values of ACC (in general, $\lesssim 10^{-8}$ ).

After assessing of the better choice for using of the parameters of the various numerical routines and the definition of the accuracy of the integration in time, we carried out several tests to verify the physical validity of the code results.

\subsection{Energy conservation}

In the code output file DATIP, we stored values of several parameters of interest. Two of them provided very useful information on the goodness of the numerical integration. The first one is the ratio, $\epsilon_{r} / \epsilon_{i}$, between the radiation energy density at each time and the energy density corresponding to the unperturbed

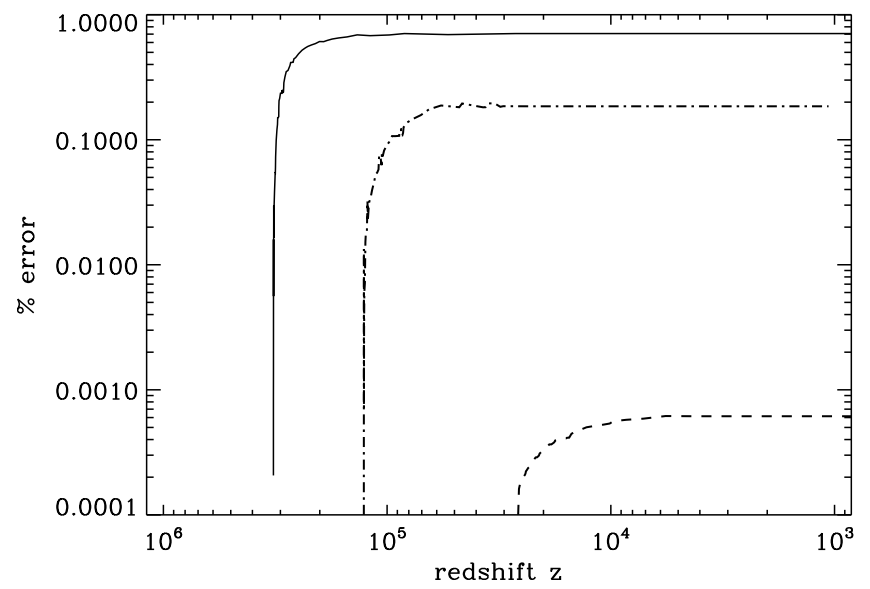

Fig. 1. Error in the energy conservation expressed in terms of relative $(\%)$ deviation from its input value of the quantity $\Delta \epsilon_{r} / \epsilon_{i}=10^{-5}$. The accuracy of the integration in time is set to ACC $=10^{-12}$ (see also the text for further details on computation). Solid, dot-dashed, and dashed lines refer to an energy injection occurred respectively at $y_{\mathrm{h}}(z) \simeq 1.5$, $0.25,0.01$. Obviously, this error further decreases improving the accuracy parameter adopted in the numerical integration.

distribution function before the distortion ${ }^{11}$ in the absence of dissipation processes. The exact energy conservation is represented by the constance of this ratio during the integration. To quantify the accuracy supplied by KYPRIX, the values of $\epsilon_{r} / \epsilon_{i}$ we stored at the start of the integration and at many times thereafter. In order to estimate the precision of the energy conservation, we define the quantity:

$\operatorname{ERR}_{\epsilon}=\frac{\left|\left(\epsilon_{r} / \epsilon_{i}\right)_{t=t_{\text {start }}}-\left(\epsilon_{r} / \epsilon_{i}\right)_{t>t_{\text {start }}}\right|}{\left(\epsilon_{r} / \epsilon_{i}\right)_{t=t_{\text {start }}}-1}$,

which gives the relative induced error in the energy conservation with respect to the initial value of $\Delta \epsilon_{r} / \epsilon_{i}$ or, more formally, the relative error induced by numerical uncertainty on the amount of fractional injected energy. Typical results obtained starting from a superposition of blackbodies are reported in Fig. 1.

Since the same absolute numerical integration error corresponds to a larger relative error for a smaller distortion, i.e. for smaller $\Delta \epsilon_{r} / \epsilon_{i}$ in these models, we could in principle expect a relative degradation of the energy conservation for decreasing distortion amplitudes. Our tests in fact indicate an increase in the relative degradation of the energy conservation for decreasing distortions when the same accuracy parameters are adopted. On the other hand, one can select them according the specific problem. For example, considering an energy injection of $\Delta \epsilon_{r} / \epsilon_{i}=10^{-5}$ occurred at $z \sim$ few $\times 10^{4}$, an accuracy equal to $\mathrm{ACC}=10^{-8}$ is fully satisfactory for a very precise calculation of the photon distribution function; while for earlier processes, such as for $z \gtrsim$ few $\times 10^{5}$, and for the same injected fractional energy, the accuracy parameter needs to be set to ACC $\leq 10^{-12}$ to assure a calculation with $\mathrm{ERR}_{\epsilon}$ less than $1 \%$. We find that for suitable choices of the integration accuracy parameters, ERR $\mathrm{ER}_{\epsilon}$ can always be kept below $\simeq 0.05 \%$ without requiring too a long computational time. Finally, we note that in some circumstances

\footnotetext{
11 For example, for a Bose-Einstein distorted spectrum with a dimensionless chemical potential $\mu_{0}$ produced by an energy dissipation with negligible photon production $\epsilon_{r} / \epsilon_{i}=f\left(\mu_{0}\right) / \varphi^{4 / 3}\left(\mu_{0}\right)$ (see e.g. Sunyaev \& Zeldovich (1970); Danese \& de Zotti (1977) and also Eqs. (8) and (9) in Burigana et al. (1991a) for the definition of $f\left(\mu_{0}\right)$ and $\varphi\left(\mu_{0}\right)$ for arbitrary values of $\mu_{0}$; for $\mu_{0} \ll 1, f\left(\mu_{0}\right) \simeq 1-1.11 \mu_{0}$ and $\varphi\left(\mu_{0}\right) \simeq$ $\left.1-1.368 \mu_{0}\right)$
} 


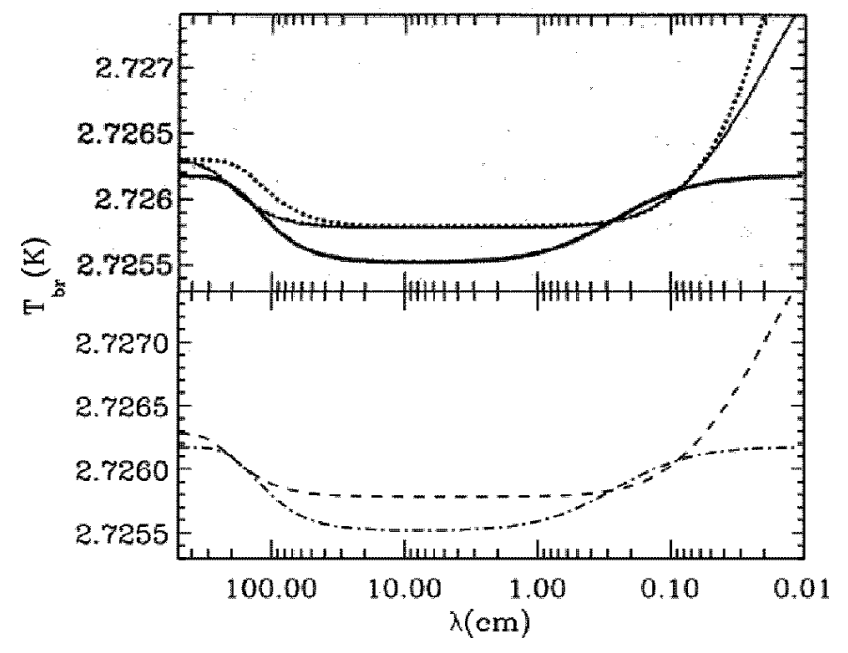

Fig. 2. Comparison between the present time solution for the CMB spectrum obtained from the old version of the numerical code (top panel; adapted from panel a of Fig. 1 in Burigana et al. 1995) and the current one (bottom panel). See the text for further details on computation parameters. Dashed (dot-dashed) line refers to an energy injection occurred at $y_{\mathrm{h}}(z) \simeq 0.01\left(y_{\mathrm{h}}(z) \simeq 0.25\right)$. In the case of the old version of the code we report also the analytical approximation (dotted line) described by a Comptonization spectrum plus a free-free distortion (see Eq. 50).

the scheme for the electron temperature evolution in the new version of KYPRIX (backward differences), different from that used in the original one (implicit scheme), could imply some small discontinuities in the evolution of the electron temperature and of $\Delta \epsilon_{r} / \epsilon_{i}$. On the other hand, we have verified that this does not affect the accuracy of the solution, because of the very small amplitude of these discontinuities (together with their localization to a very limited number of time steps) and of the corresponding energy conservation violation.

\subsection{Comparative tests}

\subsubsection{Comparing solutions}

The first kind of test is a simple comparison between the results obtained with the updated version of KYPRIX and those obtained with the original version for the same set of input parameters.

To do this, we have considered some interesting cases carried out in the past. In particular we used the input parameters adopted in (Burigana et al. 1995) where semi-analytical descriptions of the numerical solutions of the Kompaneets equation were also reported. We report here cases characterized by a specific amount of exchanged fractional energy $\Delta \epsilon_{r} / \epsilon_{i}=10^{-4}$. We started the integration from a redshift corresponding to $y_{\mathrm{h}}(z) \simeq$ 0.25 in one case and to $y_{\mathrm{h}}(z) \simeq 0.01$ in another. The input cosmological parameters are $H_{0}=50, \widehat{\Omega}_{\mathrm{b}}=0.03, k=1.68, T_{0}=$ $2.726 \mathrm{~K}$. The results given by the update version of KYPRIX are fully consistent with those reported in Burigana et al. (1995) (see Fig. 2 and note the excellent agreement between the results of the two codes). Moreover, since that paper also provided a seminalytical description of the solution of the Kompaneets equation, it is clear that the good agreement of the numerical results obtained with the original and update code represents further confirmation of the validity of the analytical description.

\subsubsection{Electron temperature behavior}

While the energy exchange between matter and radiation is led by Compton scattering (in the absence of external energy dissipation processes), electrons reach the equilibrium Compton temperature (Peyraud 1968; Zeldovich \& Levich 1970), $T_{\mathrm{e}, \mathrm{eq}}$, in a shorter time than the expansion time. For $y_{\mathrm{h}} \ll 1$, (late heating processes) Compton scattering is no longer able to significantly modify the shape of the perturbed spectrum, and the final electronic temperature $\phi_{\mathrm{f}}$ (remember that $\phi=T_{\mathrm{e}} / T_{\mathrm{r}}$ ) immediately after the decoupling is very close to $\phi_{\mathrm{eq}}=T_{\mathrm{e}, \mathrm{eq}} / T_{\mathrm{r}}$. On the other hand, for energy injections at redshifts corresponding to $y_{\mathrm{h}} \gtrsim 5$, Compton scattering can establish kinetic equilibrium between matter and radiation. This corresponds to a Bose-Einstein spectrum, with a final electron temperature given by (Sunyaev \& Zeldovich 1970; Danese \& de Zotti 1977)

$\phi_{\mathrm{f}}\left(y_{\mathrm{h}} \gtrsim 5\right)=\phi_{\mathrm{BE}} \simeq\left(1-1.11 \mu_{0}\right)^{-1 / 4}$,

where $\mu_{0}(\ll 1)$ is the usual dimensionless chemical potential. Moreover, in this case the evolution of the chemical potential, $\mu(z)$, the relation between it and the amount of fractional energy injected, $\Delta \epsilon / \epsilon_{i}$, depends on the energy injection epoch.

For the intermediate energy injection epochs, corresponding to $y_{\mathrm{h}} \lesssim 5$, the final value of $\phi$ (a function depending on $y_{\mathrm{h}}$ ) is between the values of $\phi_{\mathrm{BE}}$ and $\phi_{\mathrm{eq}}$, because the Compton scattering works to produce a Bose-Einstein like spectrum anyway (Burigana et al. 1991a). At these epochs the relation between the chemical potential and the amount of fractional injected energy injected is simply given by (Sunyaev \& Zeldovich 1970; Danese \& de Zotti 1977):

$\mu_{0} \simeq 1.4 \frac{\Delta \epsilon}{\epsilon_{i}}$

By exploiting the numerical results, a simple formula for $\phi$ can be found (Burigana et al. 1995):

$\phi_{\mathrm{f}}\left(y_{\mathrm{h}}\right)=\frac{k}{5} \frac{5-y_{\mathrm{h}}}{k+y_{\mathrm{h}}}\left(\phi_{\mathrm{eq}}-\phi_{\mathrm{BE}}\right)+\phi_{\mathrm{BE}} ;$

here $k=0.146$ is a constant derived from the fit. Moreover, this expression represents an accurate description of the evolution of $\phi$ for any value of $y_{\mathrm{h}}$. In fact, for a value of $y\left(y<y_{\mathrm{h}}\right)$ they find

$\phi\left(y, y_{\mathrm{h}}\right)=\phi_{\mathrm{f}}\left(y_{\mathrm{h}}-y\right)$.

In these cases, as in many situations of interest, the perturbed spectrum of the radiation (immediately after the heating process) is described by a superposition of blackbodies and the equilibrium temperature is given by (Zeldovich \& Sunyaev 1969; Zeldovich et al. 1972; Burigana et al. 1995)

$\phi_{\mathrm{eq}} \simeq(1+5.4 u) \phi_{i}$

where $\phi_{i}=T_{i} / T_{\mathrm{r}}=\left(1+\Delta \epsilon / \epsilon_{i}\right)^{-1 / 4} \simeq 1-u$ and the Comptonization parameter $u$ could be related to the amount of fractional energy exchanged by (Zeldovich \& Sunyaev 1969; Zeldovich et al. 1972; Burigana et al. 1995):

$u \simeq(1 / 4) \Delta \epsilon / \epsilon_{i}$.

Equations (45) and (46) can be used to test the behavior of the electron temperature during the numerical integration of the Kompaneets equation carried out with the new code version. With the increase in the time variable, the values of $\phi$ are saved into the file DATIP, from the initial time step to the final one. The upper panel of Fig. 3 shows the two behaviors 


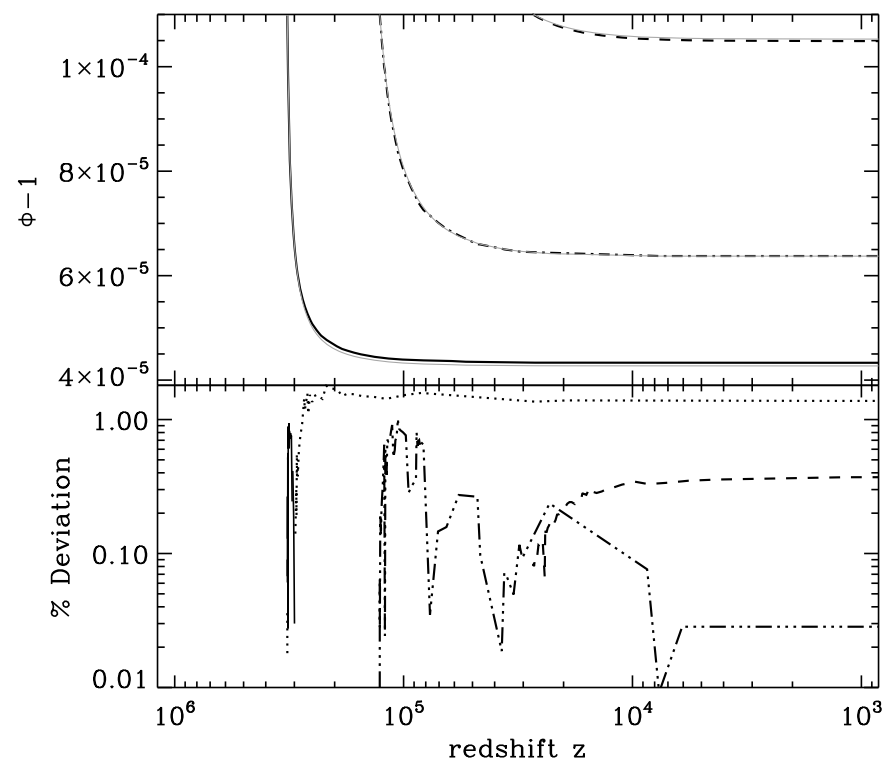

Fig. 3. Top panel: evolution of $\phi$ as derived from the numerical code. The parameters adopted for the computation are the same as in Fig. 1, as well as the adopted kinds of lines. We also report for comparison the analytical results obtained from Eq. (45) (thin solid lines). Bottom panel: relative differences between the analytical results and the numerical results expressed in terms of $\left(\phi_{\text {analytical }}-\phi_{\text {numerical }}\right) /\left(\phi_{\text {numerical }}-1\right)$. In absolute value they are $\$ 1 \%$ at each time. The lines are the same as in Fig. 1, but a solid line is replaced by dots when the above relative difference is negative.

of $\phi$ (the numerical one and the analytical expression given by Eqs. (45) and (46)), while the bottom panel displays their relative difference.

This test is of particular importance for checking the validity of the results because of the crucial role of $\phi$ in the Kompaneets equation. As remembered in the previous section, in the new version of the code a different scheme is used than implemented in the original version. Verifying of the very good agreement of these behaviors of $\phi$ further supports the equivalence of the two code versions and their reliability. In particular, it confirms that the new adopted numerical scheme for the evolution of $\phi$, in principle less stable than the implicit scheme implemented in the code original version, does not affect the validity of the solution.

\subsection{Free-free distortion}

As already discussed in Sunyaev \& Zeldovich (1970), accurate measures of the CMB spectrum in the Rayleigh-Jeans region could provide quantitative informations about the thermal history of the Universe at primordial cosmic epochs. On the other hand, photon production processes (mainly radiative Compton at earlier epochs and bremsstrahlung at later epochs) work to reduce the CMB spectrum depression at long wavelengths (see Danese \& de Zotti 1980) since they try to establish a true (Planckian) equilibrium. For $z<z_{\mathrm{p}}$ (Danese \& de Zotti 1980; Burigana et al. 1991a) with

$z_{\mathrm{p}} \simeq 2.14 \times 10^{4}\left(\frac{T_{0}}{2.7 \mathrm{~K}}\right)^{1 / 2}\left(\frac{k}{1.68}\right)^{1 / 4} \widehat{\Omega}_{\mathrm{b}}^{-1 / 2}$,

low-frequency photons are absorbed before Compton scattering moves them to higher frequencies.
In the case of small and late distortions $\left(z_{\mathrm{h}} \lesssim z_{\mathrm{p}}\right)$, a good approximation of the whole spectrum is given by (Burigana et al. 1995)

$$
\begin{aligned}
\eta(x, \tau)= & \eta_{i} \mathrm{e}^{-\left(\tau-\tau_{\mathrm{h}}\right)}+\mathrm{e}^{-\left(\tau-\tau_{\mathrm{h}}\right)} \int_{\tau_{\mathrm{h}}}^{\tau} \mathrm{e}^{\left(\tau^{\prime}-\tau_{\mathrm{h}}\right)} \frac{1}{\mathrm{e}^{x / \phi\left(\tau^{\prime}\right)}-1} \mathrm{~d} \tau^{\prime} \\
& +u \frac{x / \phi_{i} \mathrm{e}^{x / \phi_{i}}}{\left(\mathrm{e}^{x / \phi_{i}}-1\right)^{2}}\left(\frac{x / \phi_{i}}{\tanh \left(x / 2 \phi_{i}\right)}-4\right),
\end{aligned}
$$

where the index $i$ denotes the initial value of the corresponding quantity and $u$ is the Comptonization parameter. This expression provides also an exhaustive description of continuum spectral distortion generated in various scenarios of (standard or late) recombination or associated to the cosmological reionization. For an initial blackbody spectrum at dimensionless frequencies $x_{\mathrm{B}} \ll x \ll 1$, the above equation can be simplified to (Burigana et al. 1995)

$\eta \simeq \eta_{\mathrm{BB}, i}+\frac{y_{\mathrm{B}}}{x^{3}}-u \frac{2}{x / \phi_{i}}$.

Here, $y_{\mathrm{B}}$, an optical depth of the Universe for bremsstrahlung absorption (radiative Compton can be neglected at late epochs), is analogous to the Comptonization parameter, and it is given by

$$
\begin{aligned}
y_{\mathrm{B}} & =\int_{t_{\mathrm{h}}}^{t}\left(\phi-\phi_{i}\right) \phi^{-3 / 2} g_{\mathrm{B}}(x, \phi) K_{0 \mathrm{~B}} \mathrm{~d} t \\
& =\int_{1+z}^{1+z_{\mathrm{h}}}\left(\phi-\phi_{i}\right) \phi^{-3 / 2} g_{\mathrm{B}}(x, \phi) K_{0 \mathrm{~B}} t_{\exp } \frac{\mathrm{d}(1+z)}{1+z}
\end{aligned}
$$

where $x_{\mathrm{B}}$ is the frequency at which $y_{\mathrm{abs}, \mathrm{B}}=1$ (Zeldovich et al. 1972; de Zotti 1986). The dependence of the Gaunt factor (Karzas \& Latter 1961; Rybicki \& Lightman 1979; Burigana et al. 1991a) on $x$ and $\phi$ at very long wavelengths is weak: $g_{\mathrm{B}} \propto \ln (x / \phi)$.

In terms of brightness temperature, the distortions at low frequencies (at any redshift) can be written as

$\frac{T_{\mathrm{br}}-T_{\mathrm{r}} \phi_{i}}{T_{\mathrm{r}}} \simeq \frac{y_{\mathrm{B}}}{x^{2}}-2 u \phi_{i}$

where $T_{\mathrm{r}}=T_{0}(1+z)$. This approximation holds at low frequencies but not at too low frequencies, where the brightness temperature obviously approaches the electron temperature because of the extremely high efficiency of bremsstrahlung, still able to generate a Planckian spectrum at electron temperature.

To show that our numerical solution follows the behavior described by the above equation, we can compute $y_{\mathrm{B}}$ from the brightness temperature derived from the numerical solution:

$y_{\mathrm{B}} \simeq x^{2}\left(\frac{T_{\mathrm{br}}-T_{\mathrm{r}} \phi_{i}}{T_{\mathrm{r}}}+2 u \phi_{i}\right)$.

The reported numerical result (see Fig. 4) refers to a heating process corresponding to a full reionization starting at $z \simeq 20$ with $\phi=10 \times 10^{4} \mathrm{~K}$, producing a final Comptonization parameter $u \simeq 4 \times 10^{-6}$ compatible with FIRAS upper limits. As shown in Fig. $4, y_{\mathrm{B}}$ approaches an almost constant value at low frequencies. This is only correct for $\lambda>200-300 \mathrm{~cm}$, while at higher frequencies $y_{\mathrm{B}}$ is no longer almost constant (see again Fig. 4) because of the dependence of the Gaunt factor on $x$ and $\phi$ as expressed in the definition of $y_{\mathrm{B}}$. We can also write an expression describing the brightness temperature through a constant parameter $\bar{y}_{\mathrm{B}}$, derived from Eq. (54) (see Fig. 4) averaged over the 

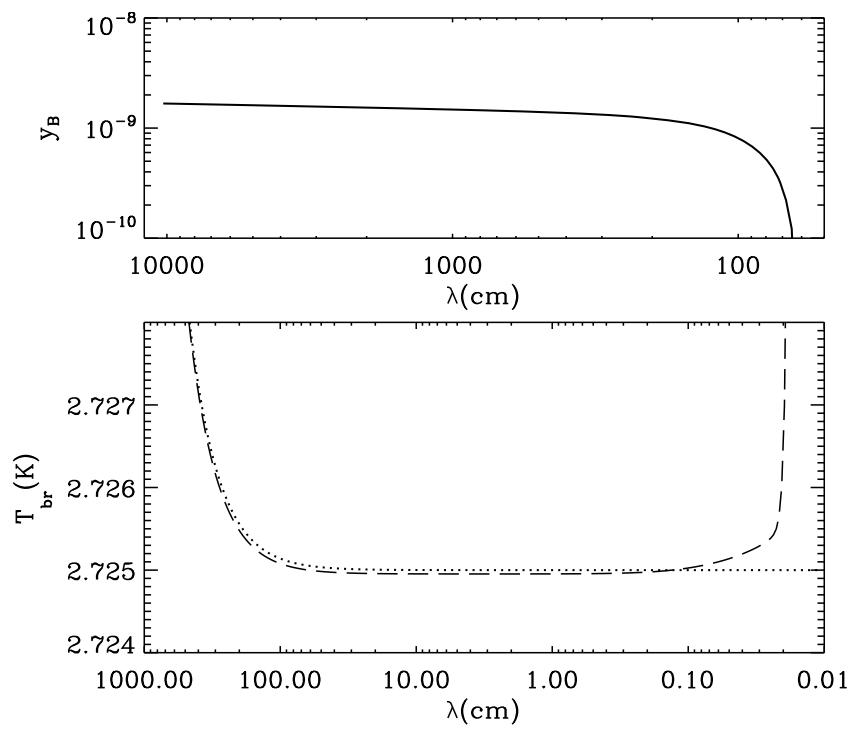

Fig. 4. Top panel: $y_{\mathrm{B}}$ as derived from Eq. (54). Bottom panel: comparison between the numerical (long dashes) solution and the analytical approximation represented by Eq. (55). See the text for further details.

range at very long frequencies before the $y_{\mathrm{B}}$ declining shown in Fig. 4, to verify the accuracy of the below expression

$T_{\mathrm{br}, y_{\mathrm{B}}}=\left(\frac{\bar{y}_{\mathrm{B}}}{x^{2}}-2 u \phi_{i}+\phi_{i}\right) \cdot T_{\mathrm{r}}$

in comparison with the numerical results. Where the Gaunt factor dependence on $x$ and $\phi$ produces a significantly varying $y_{\mathrm{B}}$ (see Fig. 4), the Comptonization decrement is more relevant than the free-free excess in determining the brightness temperature, as is evident from the good agreement of the two curves in Fig. 4. Clearly, the brightness temperature derived in this way works only up to frequencies $(\sim 100 \mathrm{GHz})$ approaching where the excess in the brightness temperature produced by the Comptonization begins (at $\sim 220 \mathrm{GHz}$, see Fig. 4).

The use of $\bar{y}_{\mathrm{B}}$ in Eq. (55) implies a slight excess $\left(\sim\left(y_{\mathrm{B}}-\right.\right.$ $\left.\left.\bar{y}_{\mathrm{B}}\right) T_{\mathrm{r}} / x^{2}\right)$ with respect to the accurate numerical results since $y_{\mathrm{B}}$ decreases with the frequency (see Fig. 4). In this representative test, the excess is $\approx 0.1 \mathrm{mK}$, but obviously its value depends on the amplitude of free-free distortion (other than on the frequency). This error is clearly negligible for analysis of current data (see e.g. Salvaterra \& Burigana 2000, 2002; Zannoni et al. 2008; Singal et al. 2009). It is likely not so relevant for analyzing future measures at $\lambda \gtrsim 1 \mathrm{~cm}$ with accuracy comparable to what is proposed for DIMES (Kogut 1996; Burigana \& Salvaterra 2003). On the contrary, it could be relevant for a very accurate analysis of future measures with precision comparable to what is proposed for FIRAS II $\lambda \lesssim 1 \mathrm{~cm}$ (Fixsen \& Mather 2002; Burigana et al. 2004; Mather 2009) or conceived for possible future long wavelength experiments from the Moon ${ }^{12,13}$ (Burigana et al. 2007). This calls for a complete frequency and thermal history-dependent treatment of the free-free distortion in the accurate analysis of future data of extreme accuracy.

\footnotetext{
12 http://wWw.Inf.infn.it/conference/moon07/Program. html

${ }^{13}$ http://sci.esa.int/science-e/www/object/index.cfm? fobjectid $=40925$
}

\section{Discussion and conclusion}

We have described the fundamental numerical approach and, in particular, the recent update to recent NAG versions of a numerical code, KYPRIX, specifically written to solve the Kompaneets equation in a cosmological context, aimed at very accurate computation of the CMB spectral distortions under quite general assumptions. The recipes and tests described in this work can be useful for implementing accurate numerical codes for other scientific purposes using the same or similar numerical libraries or for verifying the validity of different codes aimed at the same or similar problems.

Specifically, we discussed the main subdivisions of the code and the most relevant aspects about technical specifications and code implementation. After presenting the equation formalism and the boundary conditions added to the set of ordinary differential equations derived from the original parabolic partial differential equation, we gave details on the adopted space (i.e. dimensionless frequency) grid, on the output results, on the accuracy parameters, and on the integration routines. The time dependence of the ratio between electron and photon temperatures and of the radiative Compton scattering term, both introducing integral terms into the Kompaneets equation, was addressed in the specific context of the recent NAG versions by discussing the solution adopted to solve the various related technical problems.

Some of the tests carried out to verify the reliability, accuracy, and performance of the code are presented. We compared the results of the update version of the code with those obtained with the original one, reporting some representative cases, and we find an excellent agreement.

Some specific quantitative tests we reported. They indicate very good accuracy in energy conservation: for appropriate choices of the code accuracy parameters, the fractional injected energy is conserved within an accuracy better than $0.05 \%$, or, in other words, possible energy conservation violations are negligible in practice for theoretical predictions and for comparison with current and future data. The time behavior of the electron temperature is in excellent agreement with the results obtained with the original code version, in spite of the different schemes adopted to update the evolving electron temperature. These are important verifications that probe that the current implementation of the code KYPRIX circumvents the problem represented by the lost possibility of internally adapting the solver of partial differential equation to make it directly able to include integrals of the solution vector exactly at the current time step. Also, setting the accuracy parameters of the solver is now less flexible. Of course, these features imply a certain increased complexity in the code implementation, use, and in settings of code parameters. In spite of these difficulties, and thanks to better treatment of various computational steps involving integrals of functions over finite intervals, the new version of KYPRIX achieves good accuracy even in treating very small distortions.

We also described the introduction of the cosmological constant in the terms controlling the general expansion of the Universe in agreement with the current concordance model, of the relevant chemical abundances, and on the ionization history, from recombination to cosmological reionization. While an extensive discussion of various possible applications of these new features of the code, the object of future work, is not within the scope of this paper, we report here a few representative examples.

In Fig. 5 we show the effect of different treatments of the computation of the hydrogen and helium ionization fractions during the recombination epoch in the case of a relatively 


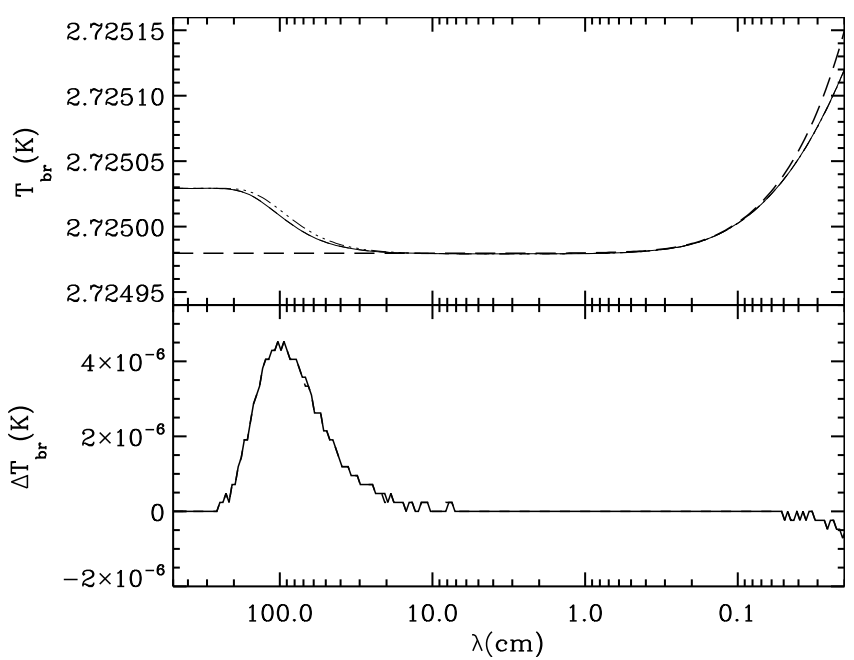

Fig. 5. Final (i.e. at $z=1090$ ) distorted spectra for different treatments of the recombination process. We assume as initial condition a Comptonization spectrum at $z=10^{4}$ characterized by $\Delta \epsilon_{r} / \epsilon_{i}=10^{-5}$. Top panel: initial condition (dashes), final spectrum in the case of instantaneous recombination at $z=1090$ following the fully ionized phase (three dots-dashes), final spectrum in the case of gradual, more realistic modelizations of the recombination process (the two different treatments give results indistinguishable in these plots). Bottom panel: difference between the final solution found in the case of instantaneous recombination and of gradual, more modelizations. See the text for further details.

late spectral distortion. We exploited three different treatments of the recombination history: an instantaneous recombination following the fully ionized phase and two gradual, more realistic modelizations of the recombination process characterized by different evolutions of ionization fractions. In one of these two cases, only the evolution of the electron ionization fraction, $\chi_{\mathrm{e}}$, was taken from the code RECFAST, while the $\mathrm{H}$ and He ionization fractions were computed solving the system of Saha equations; in the other case we directly use the complete output of the code RECFAST (see Sect. 4.3 and the caption of Fig. 5 for more details). The results in these two cases are identical in practice, while the assumption of full ionization up to $z=1090$ in the instantaneous recombination case produces a certain overestimate $(\sim 10 \%)$ of the long wavelength excess due to (essentially freefree) photon production, as well as a small amplification $(\sim 1 \%)$ of the relaxation of the initial Comptonization spectrum towards a Bose-Einstein like spectrum (obviously ineffecient, at the considered redshifts). With the adopted numerical accuracy (ACC $=$ $10^{-12}$ ) and grid points (NPTS $\left.=30001\right)$, the required CPU time on the chosen IBM platform is about $40 \mathrm{~min}$, with less than $20 \%$ differences between the three different cases.

Figure 6 reports the results derived for a particular astrophysical scenario of cosmological reionization. We adopt here the evolution of the electron ionization fraction $\chi_{\mathrm{e}}$ (the $\mathrm{H}$ and He ionization fractions were then computed to solve the system of Saha equations) and of electron temperature predicted in the suppression model presented in Schneider et al. (2008) and Burigana et al. (2008), which implies a Thomson optical depth, $\tau \simeq 0.1017$, almost in agreement with recent WMAP data. (For consistency, we adopt in these computations the same cosmological parameters used in the quoted works.) For comparison, we exploited a simple case with full ionization and a constant ratio, $\phi$, between the electron temperature and the radiation temperature that is chosen to have the same amount of energy injected into the plasma $\left(\Delta \epsilon_{r} / \epsilon_{i} \simeq 7.6 \times 10^{-7}\right)$ obtained in the above

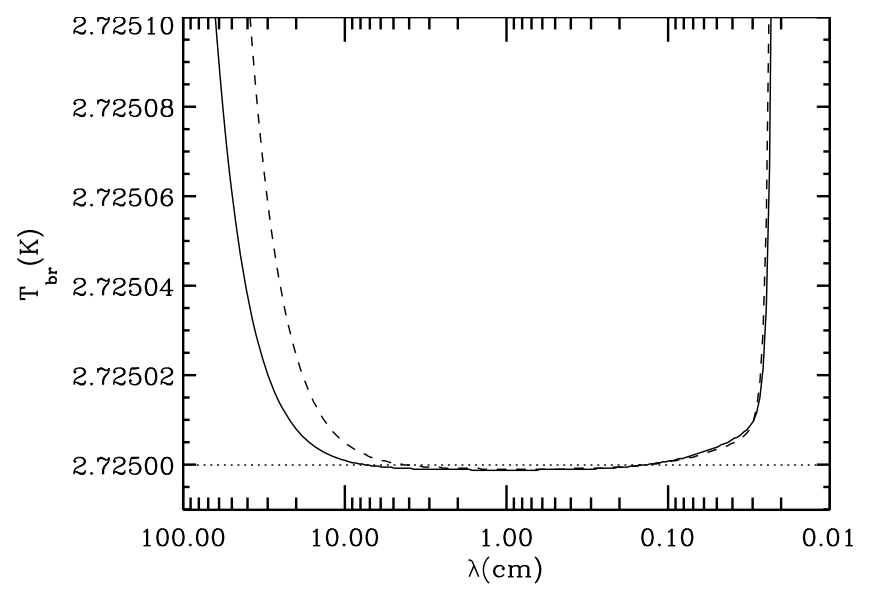

Fig. 6. Distorted spectra predicted for the considered reionization models: initial blackbody spectrum (dots) at $z=20$, almost corresponding to the beginning of the heating phase in the model, final (at $z=0$ ) spectrum in the case of constant $\phi(=147)$ (dashes), and for the realistic implementation of the ionization and thermal history in the suppression model (solid line). See the text for further details.

realistic model. As expected by construction, the two very different histories produce the same high frequency (Comptonization like) distortion, but the long wavelength region is very different because of the contribution of free-free emission ${ }^{14}$. The difference in this term in the two cases is mainly characterized by the evolution of the product $\phi^{-1 / 2} \chi_{\mathrm{e}}^{2}$ and by the free-free being more efficient at higher redshifts in the simple case than in the realistic one (see the caption of Fig. 6 for more details). With the adopted numerical accuracy $\left(\mathrm{ACC}=10^{-12}\right)$ and grid points (NPTS $=30001)$, the required CPU time on this IBM platform is a few hours for the realistic model and about one hour in the simple model with a constant $\phi$.

Finally, we focused on some properties of the free-free distortions, relevant for the long wavelength region of the CMB spectrum, by checking that the new code version, such as the original one, very accurately recovers the existing analytical approximations in their limit of validity. We also discussed the relevance of accurate computations able to improve the simple treatment based on the approximation with a frequency independent, free-free distortion parameter.

All the tests demonstrate the reliability and versatility of the new code version and its very good accuracy and applicability to scientific analysis of current CMB spectrum data and of those data, much more precise, that will be available in the future.

Acknowledgements. It is a pleasure to thank L. Danese, G. De Zotti, R. Salvaterra, and A. Zizzo for useful discussions and collaborations. Some of the calculations presented here were carried out on an alpha digital unix machine at the IFP/CNR in Milano and at the IBM SP5/512 machine at CINECA-Bologna by using some NAG integration codes. We warmly thank M. Genghini and C. Gheller for their kind assistance and technical support related to the machines used. We acknowledge the use of the code RECFAST. We acknowledge the support by the ASI contract I/016/07/0 "COFIS". We thank the anonymous referee for constructive comments.

14 In both cases, the density contrast in the intergalactic medium associated to the formation of cosmic structures has been not included. The excess at low frequencies due to the free-free distortion should be then considered as lower limit in both cases, since the computation has been performed in the "averaged density" approximation. Therefore, a correction factor $\approx\left\langle n_{\mathrm{e}}^{2}\right\rangle /\left\langle n_{\mathrm{e}}\right\rangle^{2}>1$, coming from a proper inclusion of the treatment of density contrast in the intergalactic medium, should be applied to the free-free term. 


\section{References}

Berzins, M. 1990, in Scientific Software Systems, ed. J. C. Mason, \& M. G. Cox (Chapman and Hall), 59

Berzins, M., Dew, P. M., \& Furzeland, R. M. 1989, Appl. Numer. Math., 5, 375

Burigana, C., Danese, L., \& de Zotti, G. 1991a, A\&A, 246, 49

Burigana, C., Danese, L., \& de Zotti, G. 1991b, ApJ, 379, 1

Burigana, C., de Zotti, G., \& Danese, L. 1995, A\&A, 303, 323

Burigana, C., \& Salvaterra, R. 2003, MNRAS, 342, 543

Burigana, C., Salvaterra, R., \& Zizzo, A. 2004, in Plasmas in the Laboratory and in the Universe: New Insights and New Challenges, AIP Conf. Proc., 703, 397

Burigana, C., Valenziano, L., De Rosa, A., et al. 2007, Perspectives for Future Experiments and Studies on Cosmic Background Radiation from the Moon, ASI Contract I/032/06/04, 125

Burigana, C., Popa, L. A., Salvaterra, R., et al. 2008, MNRAS, 385, 404

Chang, J. S., \& Cooper, G. 1970, J. Comput. Phys., 6, 1

Danese, L., \& Burigana C. 1994, in Present and Future of the Cosmic Microwave Background, ed. J. L. Sanz, E. Martinez-Gonzales, \& L. Cayon (Heidelberg, FRG: Springer Verlag), Lecture Notes Phys., 429, 28

Danese, L., \& de Zotti, G. 1977, Riv. Nuovo Cimento Ser., 7, 277

Danese, L., \& de Zotti, G. 1980, A\&A, 84, 364

Dew, P. M., \& Walsh, J. 1981, ACM Trans. Math. Software, 7, 295

de Zotti, G. 1986, Progr. Particle. Nucl. Phys., 17, 117

Fixsen, D. J., \& Mather, J. C. 2002, ApJ, 581, 817

Fixsen, D. J., Cheng, E. S., Gales, J. M., et al. 1996, ApJ, 473, 576

Gould, R. J. 1984, ApJ, 285, 275

Karzas, W. J., \& Latter, R. 1961, ApJS, 6, 167

Kogut, A. 1996 [arXiv: astro-ph/9607100]

Kompaneets, A. S. 1956, Zh. Eksp. Teor. Fiz., 31, 876 [Sov. Phys. JEPT, 4, 730, (1957)]

Mather, J. C. 2009, in Questions of Modern Cosmology - Galileo's Legacy, ed. M. D’Onofrio, \& C. Burigana (Springer), Sect. 5.3.1, 435
Mitchell, A. R., \& Griffiths, D. F. 1980, The Finite Difference Method in Partial Differential Equations, (UK, Chichester: Wiley)

The Numerical Algorithms Group Ltd 2009, Oxford, UK

Nolta, M. R., Dunkley, J., Hill, R. S., et al. 2009, ApJS, 180, 296

Perlmutter, S., Aldering, G., Goldhaber, G., et al. 1999, ApJ, 517, 565

Peyraud, N. 1968, Phys. Lett. A, 27, 410

The Planck Collaboration 2006, The Scientific Programme of Planck, ESASCI(2005)1 [arXiv: astro-ph/0604069]

Press, W. H., Flannery, B. P., Teukolski, S. A., \& Vetterling, W. T. 1992, Numerical Recipes (Cambridge University Press)

Riess, A. G., Filippenko, A. V., Challis, P., et al. 1998, AJ, 116, 1009

Rybicki, G. B., \& Lightman, A. P. 1979, Radiative processes in astrophysics (New York: Wiley)

Salvaterra, R., \& Burigana, C. 2000, Int. Rep. ITeSRE/CNR 270/2000, March [arXiv: astro-ph/0206350]

Salvaterra, R., \& Burigana, C. 2002, MNRAS, 336, 592

Schneider, R., Salvaterra, R., Choudhury, T. R., et al. 2008, MNRAS, 384, 1525

Seager, S., Sasselov, D. D., \& Scott, D. 1999, ApJ, 523, L1

Silk, J., \& Stebbins, A. 1983, ApJ, 269, 1

Singal, J., Fixsen, D. J., Kogut, A., et al. 2009, ApJ, submitted [arXiv:0901.0546]

Skeel, R. D., \& Berzins, M. 1990, SIAM J. Sci. Statist. Comput., 11(1), 1

Sunyaev, R. A., \& Zeldovich, Y. B. 1970, Ap\&SS, 7, 20

Tricomi, F. G. 1957, Equazioni a Derivate Parziali, ed. Cremonese, Roma

Wong, W. Y., Moss, A., \& Scott, D. 2008, MNRAS, 386, 1023

Zannoni, M., Tartari, A., Gervasi, M., et al. 2008, ApJ, 688, 12

Zeldovich, Y. B., \& Levich, E. V. 1970, Zh. Eksp. Teor. Fiz., 11, 57

Zeldovich, Ya. B., \& Sunyaev, R. A. 1969, Ap\&SS, 4, 301

Zeldovich, Ya. B., Illarionov, A. F., \& Sunyaev, R. A. 1972, Zh. Eksp. Teor. Fiz., 62, 1216 [Sov. Phys. JEPT, 35, 643]

Zizzo, A., \& Burigana, C. 2005, New Astron., 11, 1 